\title{
The spectrum of confocal endomicroscopy findings in a cystic neuroendocrine tumor of the pancreas
}

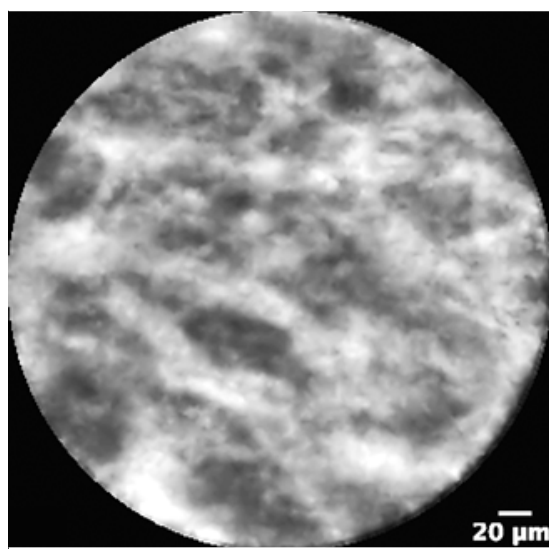

- Fig. 1 Endoscopic ultrasound with needle-based confocal laser endomicroscopy of a pancreatic cystic lesion demonstrating dark clusters of cells with surrounding gray stroma (trabeculae).

A 60-year-old man was found to have an incidental pancreatic cystic lesion during surveillance magnetic resonance imaging (MRI) for a known left renal lesion. Endoscopic ultrasound (EUS) revealed a cystic lesion $(2.6 \times 2.0 \mathrm{~cm})$ with a single thick septum in the pancreatic tail ( $\triangleright$ Video 1 ). Needle-based confocal laser endomicroscopy ( $\mathrm{nCLE}$ ) of this lesion was performed with an AQ-Flex-19 miniprobe (Cellvizio; Mauna Kea Technologies Inc., Paris, France). This demonstrated nests of cells surrounded by fibrous septa and vascularity, suggestive of a cystic pancreatic neuroendocrine tumor (PNET) ( $\mathbf{F i g . 1}$ ). A pattern of vacuolization was also observed ( $>$ Fig. 2 a; $\triangleright$ Video 1 ). Fine needle aspiration (FNA) with immunostaining of the sample obtained confirmed the diagnosis of a well-differentiated PNET.

The patient underwent distal pancreatectomy and splenectomy. Histopathology of the surgical specimen showed a well-circumscribed mixed solid-cystic lesion (\$Fig. $\mathbf{3 a ) ; ~ s y n a p t o p h y s i n ~ i m m u - ~}$ nostaining was reactive and chromatin demonstrated a salt-and-pepper pattern, consistent with a well-differentiated PNET ( Fig.3 b). Additionally, the tumor

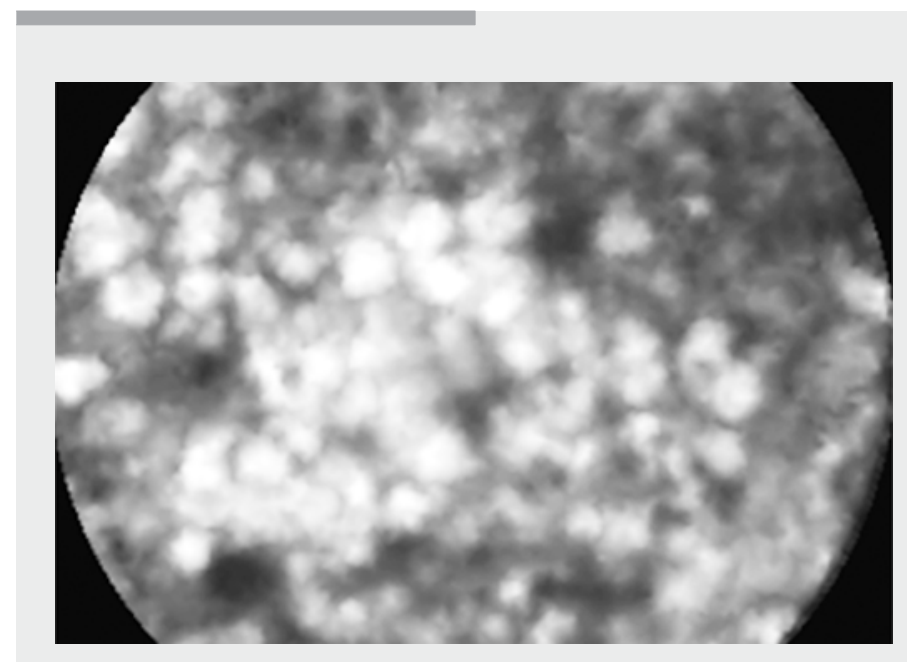

Video 1 Computed tomography, magnetic resonance, and endoscopic ultrasound imaging of a pancreatic tail lesion. Needle-based confocal laser endomicroscopy shows the established pattern of a neuroendocrine tumor with an additional novel pattern that corresponds to the histopathological appearance of the resected specimen.

cells contained hyaline globules of varying sizes within the cytoplasm ( $\mathbf{F i g} \mathbf{2}$ b). This finding correlated with the vacuolization image pattern seen on nCLE. The presence of these globules has been described in PNETs and solid pseudopapillary tumors, with the descriptive appearance of cytoplasmic vacuolization $[1,2]$. A similar pattern has been attributed to the presence of cytoplasmic lipid, which is more common in cystic NETs [3]. Napoleon et al. first described the nCLE criteria of cystic PNETs, which have been validated in resected ex vivo lesions and by independent external observers [4]. The EUS-nCLE findings so far described in cystic PNETs include a trabecular network of dark cells with surrounding fibrosis and vascularity $[4,5]$. This case presents a unique recurring feature of vacuolization that correlated with a comparable histopathological pattern. This spectrum of nCLE image patterns for cystic NETs can further assist in the differentiation of pancreatic cystic lesions.

Endoscopy_UCTN_Code_CCL_1AF_2AZ

\section{Competing interests}

None

The authors

Anjuli K. Luthra ${ }^{1}$, Christina A. Arnold ${ }^{2}$, Andrei V. Manilchuk ${ }^{3}$, Somashekar G. Krishna ${ }^{1}$

1 Division of Gastroenterology, Hepatology and Nutrition, Ohio State University Wexner Medical Center, Columbus, Ohio, USA

2 Department of Pathology, Ohio State University, Columbus, Ohio, USA

3 Division of General and Gastrointestinal Surgery, Ohio State University Wexner Medical Center, Columbus, Ohio, USA

Corresponding author

\section{Somashekar G. Krishna, MD, MPH}

395 W. 12th Avenue, 2nd floor, Division of Gastroenterology, Hepatology and Nutrition, Columbus, Ohio, USA

Fax: +1-614-293-8518

sgkrishna@gmail.com 

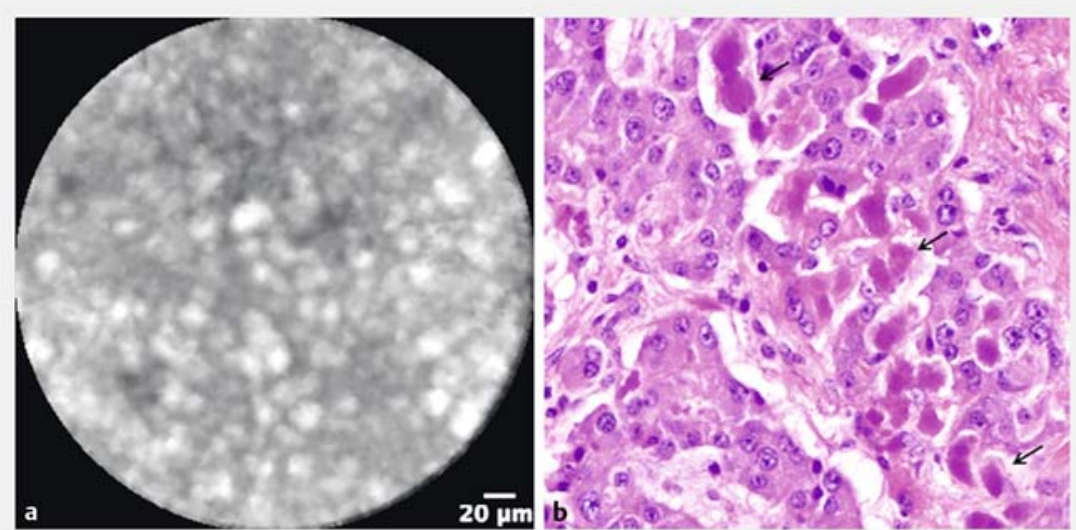

- Fig. 2 The pancreatic cystic lesion seen on: a endoscopic ultrasound needle-based confocal laser endomicroscopy showing a unique pattern of vacuolization intermixed with dark nests of cells; b hematoxylin and eosin staining of the resected specimen (magnification $\times 400$ ) showing intracytoplasmic globules (arrows) within the cords of tumor cells. Salt-and-pepper type chromatin, characteristic of neuroendocrine neoplasms is also seen.
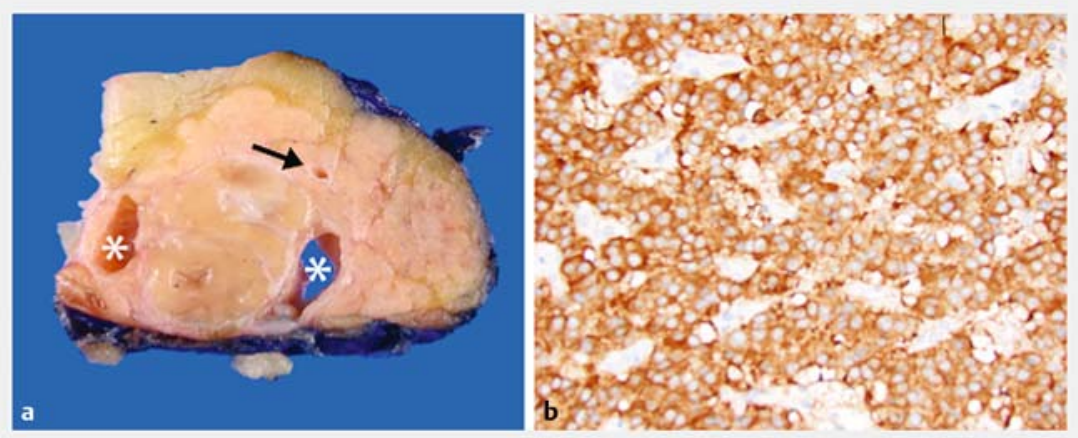

- Fig.3 Appearance of the resected specimen: a on gross examination, showing a pancreatic tail mass consisting of collapsed cystic areas (asterisks) not in communication with the pancreatic duct (arrow); b on immunostaining with synaptophysin, showing diffuse reactivity of the tumor cells, supporting the diagnosis of a well-differentiated neuroendocrine tumor. Chromogranin was also reactive (not shown); Ki67 was $3 \%$ and zero mitoses were seen per 10 high-power fields, in keeping with a WHO grade 2 neuroendocrine tumor.

\section{References}

[1] Meriden Z, Shi C, Edil BH et al. Hyaline globules in neuroendocrine and solid-pseudopapillary neoplasms of the pancreas: a clue to the diagnosis. Am J Surg Pathol 2011; 35: 981-988

[2] Levy GH, Finkelstein A, Harigopal M et al. Cytoplasmic vacuolization: an under-recognized cytomorphologic feature in endocrine tumors of the pancreas. Diagn Cytopathol 2013; 41: 623-628

[3] Fryer E, Serra S, Chetty R. Lipid-rich ("clear cell") neuroendocrine tumors of the pancreas in MEN I patients. Endocr Pathol 2012; 23: $243-246$

[4] Krishna SG, Brugge WR, Dewitt JM et al. Needle-based confocal laser endomicroscopy for the diagnosis of pancreatic cystic lesions: an international external interobserver and intraobserver study (with videos). Gastrointest Endosc 2017; 86: 644 654.e2

[5] Napoleon B, Lemaistre Al, Pujol B et al. In vivo characterization of pancreatic cystic lesions by needle-based confocal laser endomicroscopy (nCLE): proposition of a comprehensive nCLE classification confirmed by an external retrospective evaluation. Surg Endosc 2016; 30: 2603-2612

\section{Bibliography}

DOI https://doi.org/10.1055/a-0667-8978

Published online: 14.8.2018

Endoscopy 2018; 50: E323-E324

(c) Georg Thieme Verlag KG

Stuttgart · New York

ISSN 0013-726X

\section{ENDOSCOPY E-VIDEOS \\ https://eref.thieme.de/e-videos}

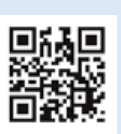

Endoscopy E-Videos is a free access online section, reporting on interesting cases and new techniques in gastroenterological endoscopy. All papers include a high quality video and all contributions are freely accessible online.

This section has its own submission website at https://mc.manuscriptcentral.com/e-videos 